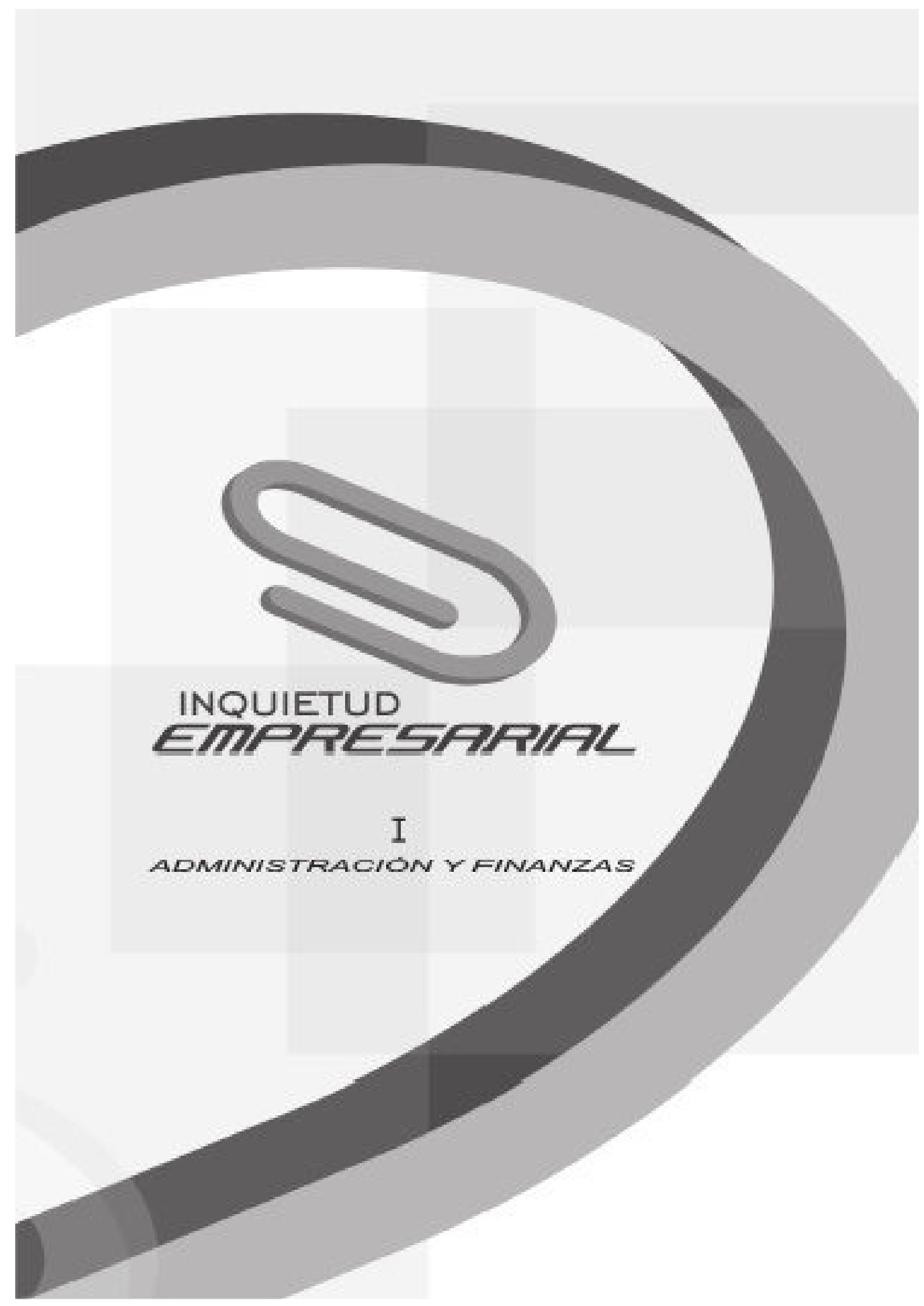




\title{
EFECTOS MEDIOAMBIENTALES EN UNA CONTABILIDAD FINANCIERA DENTRO DE UNA PYME, ESTUDIO DE CASO'.
}

\author{
ENVIRONMENTAL EFFECTS ON FINANCIAL ACCOUNTING IN AN SME, CASE \\ STUDY
}

\author{
Omar García Jiménez ${ }^{2}$ \\ Claudia Yazmin Medina Contreras ${ }^{3}$
}

Para citar este artículo: García, O. y Medina, C. (2013). "Efectos medioambientales en una contabilidad financiera dentro de una pyme, estudio de caso". Revista Inquietud Empresarial. Vol. XIII (2), p.p. 11-30

Fecha de recepción: 05 de septiembre de 2013

Fecha de Aceptación: 13 de noviembre de 2013

\begin{abstract}
1 Artículo de investigación científica y tecnológica como resultado del proyecto de investigación "La inclusión de los efectos medioambientales en la contabilidad financiera como parte integral de la Responsabilidad Social Empresarial: Un estudio de caso en Pymes del sector comercial y de servicios", adelantado en el Tecnológico de Estudios Superiores del Oriente del Estado de México (TESOEM).

2 Contador Público Certificado, Maestro en Ciencias en Administración Profesor del área de Posgrado en Finanzas del Tecnológico de Estudios Superiores del Oriente del Estado de México. omar24_ga@yahoo.com.mx

3 Estudiante de Contaduria Pública del Tecnológico de Estudios Superiores del Oriente del Estado de México (TESOEM). clauz_ztreyitaz@hotmail.com.
\end{abstract}




\title{
RESUMEN
}

Uno de los principales retos que se presenta dentro de las Pequeñas y Medianas Empresas (Pymes) es tratar de cuantificar los efectos del medio ambiente que rodean o circunscriben a la misma y por lo tanto registrar en una contabilidad tradicional. El impacto ambiental una vez que se logra cuantificar y registrar dentro de los Estados Financieros de la Pyme objeto de estudio, obtiene un valor económico incluso superior a su valor en libros y con ello una serie de probables beneficios como una certificación, acceso a créditos bancarios y principalmente la aceptación por parte de la comunidad. El objetivo de este trabajo es hacer una propuesta a los dueños de la empresa Serví Pavos de México, S.A. de C.V. de incorporar los efectos o beneficios de los impactos ambientales dentro de una contabilidad financiera con enfoque ambiental, es decir obtener un fin lucrativo lícito pero con un enfoque realmente sostenible. El trabajo de investigación se realizó mediante un estudio cualitativo basado en un cuestionario el cual se aplicó a diez accionistas de la empresa, al final se encuentra que la organización no reconoce los efectos del medio ambiente dentro de su contabilidad financiera, así como la necesidad de presentar una propuesta para su incorporación.

\section{PALABRAS CLAVE}

Pyme, Contabilidad, Medio ambiente, Beneficios adicionales, Desarrollo sostenible.

\begin{abstract}
One of the main challenges that arise within the SME is trying to quantify the effects of the environment that surround or confined to it and therefore to register in a traditional accounting. Environmental impact once does quantify and register within the financial States of the SME study object, obtained even greater than its value in books and thus economic value a series of probable benefits as a certification, access to bank credit and mainly the acceptance by the community. The objective of this work is to make a proposal to the owners of this SME incorporate the effects or benefits of environmental impacts within a financial accounting with environmental approach, to obtain a lawful lucrative purpose, but with a truly sustainable approach. The research was conducted through a qualitative study based on a questionnaire which was applied to ten shareholders of the company, eventually found that the organization does not recognize the effects of the environment within their financial accounting and the need for submit a proposal for inclusion.
\end{abstract}

\section{KEY WORDS}

Smes, accounting, environment, additional benefits, sustainable development. 


\section{INTRODUCCIÓN}

Algunos de los supuestos de las Pymes se refieren a la simplicidad en la toma de decisiones, bajos costos operativos y organizacionales, por citar algunos ejemplos. Ante dicha situación, no resulta extraño que los propietarios o dueños de la empresa se enfoquen en la operación diaria de la organización, sin planificar su futuro. "A veces no se tiene la preparación o el tiempo necesarios, pero generalmente no se percibe el valor de disponer de un plan de acción y por ello no se asignan los recursos requeridos". Debernardo y Hurtado (2010, p. 25).

La falta de información o la falta de políticas y procedimientos de control ambiental no se cuantifican o registran dentro de los sistemas contables. Es por ello que dentro de este tipo de organizaciones no se reconozca los impactos ambientes, los costos o inversiones necesarios para mitigar dicho impacto.

Por otro lado, una vez que se logran implementar las acciones necesarias para reducir el deterioro a la flora y fauna de la comunidad, se obtienen algunos beneficios adicionales y no necesariamente corresponden con una certificación por ser empresa socialmente responsable (ESR), sino que va más allá de la misma, al tratar de medir los beneficios futuros que se obtendrían por ejemplo al plantar árboles dentro de la organización; el tratamiento del uso del agua; el uso de paneles solares o empleo de energía eólica, etc., ya que se destina una inversión en activos biológicos así como un conjunto de acciones necesarias para lograr este objetivo. Con ello se lograría no impactar al medio ambiente dentro de la comunidad, esto traería un beneficio dentro de la sociedad, pero lo más importante es tratar de medir estos beneficios dentro de la organización y por supuesto incorporarlos dentro de los estados financieros, situación altamente compleja, al no resultar sencillo medir este beneficio con exactitud, debido a los beneficios futuros, ya que depende del tiempo en el cual se van a ver reflejados estos efectos positivos y como la población será beneficiada.

El estudio de caso que se plantea es a partir de una Pyme ubicada en el Estado de México, la cual no considera el efecto negativo que provoca al medio ambiente, por lo tanto, se sugiere que la empresa emplee las acciones necesarias así como las inversiones que se requieran en la medida de la posible para mitigar este efecto, y por lo tanto se hace también necesario que se registren las inversiones en activos biológicos así como los beneficios futuros dentro de los principales estados financieros.

La problemática central consiste en la inexistencia de un método que cuantifique y registre el impacto ambiental dentro de la información financiera de la empresa. La investigación se realizó con una metodología de estudio de caso, para examinar el fenómeno del porque no se registran los efectos del medio ambiente en una contabilidad tradicional. Tiene una finalidad exploratoria, descriptiva y explicativa. El objetivo primordial de la investigación y siguiendo a Yin (1989), referente a las metas que puede tener la investigación de estudio de caso, es la de contribuir a la validación de los estudios efectuados. La unidad de investigación es en una Pyme denominada Serví Pavos de México, S.A. de C.V. También se hará uso del Método comparativo al mostrarnos cifras para determinar sí se está cumpliendo con la normatividad ambiental.

Es importante señalar que el objetivo de este trabajo es verificar si la empresa sujeto a estudio registra los efectos medioambientales en su contabilidad financiera, más que construir alguna teoría o explicar los efectos medioambientales. 
Para ello el trabajo está organizado de la siguiente manera: Primero se revisa la literatura existente sobre medio ambiente, responsabilidad social empresarial para soportar una hipótesis. Enseguida se explica el método de investigación y se aplica el constructo para levantar la información. Finalmente se presentan las conclusiones o hallazgos obtenidos.

\section{Referentes teóricos}

\section{Actualización de contabilidad medioambiental}

Antes de entrar en el concepto de contabilidad ambiental, es pertinente mencionar que la contabilidad ambiental es vista ahora como un elemento esencial de la responsabilidad ambiental de cualquier organización (Gray y Bobbington, 2006).

La contabilidad ambiental se refiere al reconocimiento y revelación de los costos que generan los efectos medioambientales dentro de un sistema contable, para poder conocer los beneficios de dichos efectos, para la toma de decisiones. (Herrerías, 2009)

La contabilidad financiera mide el consumo humano a partir de los recursos de capital, y la contabilidad ambiental está orientada a la cuantificación y registro de las medidas establecidas para la protección del medioambiente de los recursos de capital natural que juegan dentro del proceso de producción.

Es importante que al iniciar la aplicación de criterios de contabilidad ambiental en una empresa se realicen los siguientes pasos:

- La empresa debe establecer políticas para la protección del medio ambiente.

- Crear planes y estructurar las políticas en acciones.

- Establecer los razonamientos de cuantificación y medición.

- Supervisar las acciones y los resultados obtenidos.

- Informar permanentemente de los logros alcanzados a los propietarios, al gobierno y a la comunidad.

La contabilidad ambiental tiene desafíos que enfrentar como son:

- Mejorar las normas y registros contables en materia ambiental.

- Contribuir a las prácticas ambientales por medio de la administración.

- Contribuir a descontaminar los balances que se emiten sin tomar en cuenta los efectos ambientales.

Hoy en día la protección al medio ambiente es una oportunidad de éxito en los negocios por lo cual es importante tomarlo en cuenta en la gestión empresarial, pero sin perder de vista los propósitos de la empresa como es el nivel de competitividad que se desea alcanzar, es importante destacar que la implementación de una contabilidad de tipo ambiental no solo se debe implantar a partir de una certificación o 
como elemento de mercadotecnia, sino que debe promover y concientizar el uso y protección al medio ambiente como una necesidad social. ${ }^{4}$

\section{- Gestión Ambiental}

Esta gestión busca establecer actividades a desarrollar a favor del medio ambiente, así como quienes serán los responsables de su ejecución, en las que se debe poseer dinamismo y flexibilidad para adaptarse a los cambios que puede sufrir el medio ambiente en un futuro inmediato.

La gestión ambiental, se señala como una de las actividades más importantes relacionada con cualquier emprendimiento empresarial (Iturria (2012).

Antonius (1999) revela que "el gerenciamiento ambiental puede ser conceptuado como la integración de sistemas y programas organizacionales”, que permitan:

a) El control y la reducción de los impactos en el medio ambiente.

b) El cumplimiento de las leyes y normas ambientales.

c) El desarrollo y uso de tecnologías apropiadas para minimizar o eliminar residuos industriales.

d) El monitoreo y evaluación de los procesos y parámetros ambientales.

e) La eliminación o reducción de los riesgos al medio ambiente y al hombre.

f) La utilización de tecnologías limpias (clean technologies), buscando minimizar los gastos de energía y materiales.

g) La mejora del relacionamiento con la comunidad y el gobierno.

h) La anticipación de cuestiones ambientales que puedan causar problemas al medio ambiente y, particularmente, a la salud humana.

Cuando la empresa toma la decisión de desarrollar la gestión ambiental puede desarrollar su propio sistema o implementar un sistema que le permita obtener la certificación por un tercero.

Un Sistema de Gestión Ambiental identifica las políticas y procedimientos que realiza la empresa para lograr una gestión efectiva. Por lo cual es sujeta a evaluaciones de impacto ambiental, ya que se adquiere el compromiso de cumplir con la normatividad en cuanto al comportamiento ambiental.

Existen diferentes normas referidas a los Sistemas de Gestión Ambiental (SGA), siendo las de mayor difusión y aplicación las siguientes:

- Reglamento 1836/93 de la Unión Europea, conocido como reglamento Eco-Management and AuditScheme, o Reglamento Comunitario de Ecogestión y Ecoauditoría (EMAS). Este Reglamento define a los SGA como "aquella parte del sistema general de gestión que comprende la estructura organiza-

\footnotetext{
4 En nuestra actividad como profesionales y durante nuestra preparación como tales, encontramos muchas áreas de estudio y análisis, en especial sobre aspectos contables, base de la formación de un contador. De esta manera hemos descubierto que la contabilidad es una técnica de por si evolutiva, conociendo y analizando una serie de áreas o ramas que la complementan, es decir contabilidad es una técnica de por si evolutiva, conociendo y analizando una serie de áreas o ramas que la complementan, es decir,
formas específicas de tratamiento que le dan ciertas operaciones y que requieren uniformidad dadas las caracteristicas particulares que tienen, por ejemplo: contabilidad forestal, agrícola, etc.
} 
tiva, las responsabilidades, las prácticas, los procedimientos, los procesos y los recursos para determinar y llevar a cabo la política ambiental".

- Serie de normas dela Organización Internacional de Normalización (ISO) 14000, de aplicación internacional, define al SGA como "la parte del sistema de gestión general que incluye la estructura organizativa, las actividades de planificación, las responsabilidades, las practicas, los procedimientos, los procesos y los recursos para desarrollar, implementar, realizar, revisar y mantener la política ambiental".(UNIT-ISO 14001)

El éxito en la implementación de un Sistema de Gestión Ambiental se basa en principios básicos entre los que destacamos como fundamental, el compromiso de la dirección superior con relación al mejoramiento del desempeño de la organización, donde se establezcan claramente los objetivos y metas ambientales, así como un necesario involucramiento coordinado y responsable en todos los niveles de la estructura organizacional. De acuerdo con Epstein (2003) "la administración ambiental corporativa mejorada no surge como consecuencia del mejor uso de los modelos existentes. Muchas empresas pueden ahorrar cientos de pesos en costos corporativos y también reducir los impactos ambientales”. (2003, p. XXXVIII).

\section{- La responsabilidad de las empresas}

La responsabilidad de una empresa significa asumir la rendición de cuentas de una serie de hechos institucionales desde una actitud ética. Es un compromiso que se adquiere al identificar los efectos que se sufren en el mundo y así poder dar una solución a los problemas que se enfrentan y cumplir con lo pactado. En ese sentido, entendemos que la responsabilidad social no es sólo de las empresas, sino también del gobierno y de la sociedad (Herrerías, 2011).

Por lo cual las empresas públicas como las privadas tiene el compromiso de realizar actividades para ser empresas socialmente responsables.

Para que una empresa pueda ser socialmente responsable tiene que llevar a cabo el cumplimiento de las obligaciones jurídicas, mayores inversiones en el capital humano, el entorno y las relaciones con los interlocutores.

Para ello en Centro Mexicano de la Filantropía (s.f), organismo fundado en 1988 de carácter privado, indica que una empresa responsable es aquella que tiene como compromiso consciente y congruente de cumplir con los objetivos de la empresa con la participación de todos los colaboradores pero también aplicando los valores y respetando el medioambiente y a las comunidades.

\section{- Beneficios}

A partir de lo anterior, podemos afirmar que la sustentabilidad organizacional y la responsabilidad social deben ser parte de la filosofía empresarial e imagen corporativa, toda vez que:

- Genera un sistema de alerta anticipada de posibles riesgos económicos, sociales o ambientales a los que el negocio está expuesto; se constituye en una herramienta de autodiagnóstico para sus procesos internos.

- Ayuda a la reducción de costos en cumplimiento de nuevas regulaciones ambientales y laborales, como resultado de la aplicación de criterios de ecoeficiencia, esto es, producir más con menos recursos y menos contami- 
nación.

- Mejora el acceso a capital gracias al fortalecimiento y transparencia en las relaciones con proveedores de capital e inversionistas.

- Contribuye a la innovación de productos y/o servicios e incrementa la lealtad de los consumidores.

- Motiva a empresarios y trabajadores a asumir el compromiso de desempeñarse hacia el logro de las metas del negocio (Mercado y García, 2013).

Diferentes tipos de contabilidad medioambiental

En la contabilidad medioambiental se deben de considerar, el siguiente cuadro corresponde a una clasificación que aparece en un documento de la Enviromental Protection Agency (s.f), (EPA) de los Estados Unidos:

Tabla 1. Diferentes tipos de contabilidad

\begin{tabular}{|c|c|c|}
\hline $\begin{array}{c}\text { Tipos de contabilidad } \\
\text { medioambiental }\end{array}$ & Enfoque & Dirigido a usuario: \\
\hline Contabilidad nacional & $\begin{array}{c}\text { Macroeconómico, economía } \\
\text { nacional }\end{array}$ & Externo \\
\hline Contabilidad financiera & La empresa & Externo \\
\hline $\begin{array}{c}\text { Contabilidad gerencial o de } \\
\text { costos }\end{array}$ & $\begin{array}{c}\text { La empresa, departamentos, } \\
\text { línea de producción, etc. }\end{array}$ & Interno \\
\hline
\end{tabular}

\footnotetext{
Fuente: Herrerías (2009).
}

La contabilidad nacional está referido a los recursos naturales de la nación de los cuales se hace uso de ellos sean renovables o no, ya sea en unidades físicas o monetarias.

La contabilidad financiera es la presentación del impacto en el medioambiente en los estados financieros, conforme a las Financial Accounting Standards Board (FASB).

La contabilidad gerencial es analizar los efectos negativos en el medioambiente al cumplir con los objetivos de la empresa y tomar decisiones con el impacto que se tiene interna como externamente que podrían modificar los costos de dichos efectos en la producción.

\section{- Normatividad ambiental}

"En México, la normatividad ambiental encuentra su base en la Constitución Política. De ésta se derivan las diversas leyes, reglamentos y normas que rigen el país. Las Normas Oficiales Mexicanas (NOMs), son el instrumento jurídico que obliga a cumplir las especificaciones que determina la autoridad federal" (Normatividad ambiental, s.f). En materia de calidad del aire, la normatividad está determinada particularmente por la Secretaría de Salud, y por la Secretaría de Medio Ambiente y Recursos Naturales, SEMARNAT. Ambas Secretarías han desarrollado NOMs enfocadas a la protección de la salud de la población y a la medición de los 
contaminantes, entre otras contamos con:

La Procuraduría de Protección al Ambiente del Estado de México (PROPAEM) las disposiciones contenidas en las disposiciones jurídicas siguientes:

Ley general del equilibrio ecológico y la protección ambiental.

Ley General de la Prevención y Gestión Integral de Residuos

Código para la Biodiversidad del Estado de México

Reglamento de la Ley General del Equilibrio Ecológico y la Protección al Ambiente, en materia de:

- Registro de emisores y transferencia de contaminantes.

- Prevención y control

- Evaluación del impacto ambiental

- Áreas naturales protegidas

- Auditoría ambiental

- Ordenamiento ecológico

Reglamento de la Ley General para la Prevención y Gestión Integral de Residuos

Reglamento del Libro Segundo del Código para la Biodiversidad del Estado de México

Reglamento del Libro Cuarto del Código para la Biodiversidad del Estado de México

Reglamento del Libro Quinto del Código para la Biodiversidad del Estado de México

Normas Técnicas estatales ambientales

Existen diversas Normas Técnicas Ambientales, que ha publicado el Gobierno del Estado de México, con el propósito de fomentar la preservación y protección del medio ambiente, las cuales rigen diversas actividades humanas, al aplicarse en diferentes procesos productivos, comerciales y de servicios como son:

- NTEA-001-SEGEM-AE-2003: que establece los requisitos para el almacenamiento, tratamiento y disposición final de los residuos orgánicos generados en centros de sacrificio de animales para el consumo humano.

- NTEA-002-DS-2009: que regula la exploración, explotación y transporte de minerales no concesionables en el Estado de México.

- NTEA-003-SEGEM-2004: que establece las especificaciones para la prevenir y controlar la contaminación visual.

- NTEA-004-SMA-DS-2006: establece las especificaciones de protección ambiental para las etapas de selección del sitio, construcción y remodelación de estaciones de servicio (gasolineras), en territorio del Estado de México. 
- NTEA-005-SMA-RN-2005: establece las especificaciones y criterios que deben observarse para el desarrollo de acciones y usos compatibles sustentables en las áreas naturales protegidas del Estado de México.

- NTEA-006-SMA-RS-2006: establece los requisitos para la producción de los mejoramientos de suelos elaborados a partir de residuos orgánicos.

- NTE-007-007SMA-DS-2006: establece los requisitos para la elaborar el índice metropolitano de la calidad del aire.

- NTEA-008-SMA-DS-2007: establece las especificaciones de protección ambiental durante las etapas de selección del sitio y construcción de estaciones de gas L.P. para carburación en el territorio del Estado de México.

- NTEA-009-SMA-RN-2008: establece las especificaciones y criterios que deben observarse para el uso, conservación y restauración de suelos en áreas naturales protegidas en el Estado de México.

- $\quad$ NTEA-010-SMA-SR-2008: establece los requisitos y especificaciones para la instalación. Operación y mantenimiento de la infraestructura para el acopio, transferencia, separación y tratamiento de residuos sólidos urbanos y de manejo especial, para el Estado de México.

- NTEA-011-SMA-DS-2008: establece los requisitos para el manejo de los residuos de la construcción para el Estado de México.

- NTEA-012-SMA-DS-2009: establece las especificaciones de protección ambiental para la selección, preparación del sitio, construcción y operación del proyecto de vivienda en el territorio del Estado de México.

- NTEA-013-SMA-RS-2011: establece las especificaciones para le separación en la fuente de origen, almacenamiento separado y entrega separada al servicio de recolección de residuos sólidos urbanos y de manejo especial para el Estado de México.

\section{Metodología de la Investigación. (Serví Pavos de México, S.A. de C.V. Estudio de caso)}

\section{- Antecedentes}

Si bien existen pocos datos cuantitativos sobre el valor económico (y social) total de la información de costos ambientales totales, la Agencia de Protección Ambiental (E.P.A.) de Estados Unidos identifica algunos beneficios claves de obtener, administrar y utilizar esta información dentro de la empresa, ellos son:

$>$ Muchos costos ambientales se pueden reducir significativamente o eliminar como resultado de decisiones de negocios, las cuales van desde cambios operacionales y de custodia, hasta inversión en tecnologías de procesos verde, hasta el rediseño de procesos / productos. Muchos costos ambientales (ejemplo: desechos de materias primas) pueden proporcionar ningún valor añadido a un proceso, sistema, o producto.

> Los costos ambientales (y por consiguiente el potencial ahorro de costos) se 
pueden oscurecer en las cuentas generales de gastos.

$>$ Han descubierto que los costos ambientales se pueden eliminar generando ingresos mediante la venta de desechos o asignaciones transferibles de contaminación (es decir la transferencia de los permisos de contaminación), o mediante licencias de tecnologías limpias.

$>$ La mejor administración de los costos ambientales puede dar como resultado un desempeño ambiental mejorado y beneficios significativos para la salud humana así como éxito en los negocios.

$>$ El entendimiento de los costos ambientales y del desempeño de procesos y productos puede promover un costeo y una fijación de precios más exactos y puede ayudar a las empresas en el diseño de procesos, productos, y servicios más preferibles ambientalmente y por consiguiente darle una ventaja competitiva a la empresa.

$>$ La contabilidad de costos y desempeño ambientales puede dar soporte al desarrollo y operación de un sistema general de administración ambiental de una empresa. Tal sistema constituirá una necesidad para las compañías vinculadas con las obligaciones de comercio internacional por el estándar de consenso internacional ISO 14001, desarrollado por la International Organization of Standarization.

Dichos costos se podrían considerar como preventivos para aquellos que se registran de manera anticipada, corrientes cuando son reconocidos en el periodo en el que se incurrió en dichos costos o también se pueden registrar como un activo diferido.

\section{- Reconocimiento de los costos ambientales}

El reconocimiento de los costos ambientales se debe de hacer en provisiones, pasivos contingentes y activos contingentes de acuerdo con las Normas Internacionales de Contabilidad (s.f), las cuales en su boletín 37 nos menciona que para poder reconocer los costos ambientales en los estados financieros se debe hacer una provisión cuando se den las siguientes condiciones:

La empresa tenga la obligación actual (ya sea legal o efectiva) como resultado de un suceso pasado.

Es probable que la empresa tenga que desprenderse de recursos, que incorporen beneficios económicos para cancelar tal obligación.

Puede hacerse una estimación fiable del importe de la obligación.

De no cumplirse las tres condiciones indicadas, la empresa no debe reconocer la provisión.

En el estado de resultados, el gasto relacionado con la provisión puede ser objeto de presentación como una partida neta del importe reconocido como reembolso a recibir.

En algunos casos, la empresa puede no tener que responder del pago de la provisión si el tercero responsable deja de realizar el desembolso. En tal caso, la empresa no tendrá responsabilidad por tales pagos, y por tanto no se incluirán en el cálculo de la provisión. 
Para cada tipo de provisión, la empresa debe informar acerca de:

A. El valor contable al principio y al final del ejercicio

B. Las dotaciones efectuadas en el ejercicio, incluyendo también los incrementos en las provisiones existentes

C. Los importes utilizados (esto es, aplicados o cargados contra la provisión) en el transcurso del ejercicio

D. Los importes no utilizados que han sido objeto de desdotación o reversión en el ejercicio, y

E. El aumento, durante el ejercicio, en los saldos objeto de descuento, por causa de los intereses acumulados, así como el efecto que haya podido tener cualquier eventual cambio en la tasa de descuento.

\section{METODOLOGÍA}

Hipótesis: La empresa sujeta a estudio reconoce dentro de su contabilidad financiera los efectos del medio ambiente.

Variable dependiente: Contabilidad financiera. La contabilidad financiera se centra en las necesidades específicas de aquellas personas externas a la empresa que toman decisiones, entre ellos los accionistas, los proveedores, los bancos los organismos gubernamentales (Horngreen y otros, 2000).

Variable independiente: Efectos del medio ambiente. Podríamos considerar el medio ambiente como un conjunto de cosas, condiciones e influencias como clima, temperatura, relaciones con otras personas y efectos derivados de ellas. El medio ambiente es todo, es el entorno vital (Barraza y Gómez, 2005)

El trabajo de investigación se realizó mediante un estudio cualitativo basado en un cuestionario con diez preguntas referentes a la que si la empresa reconoce o registra dentro de su contabilidad los efectos medioambientes, se obtuvieron los siguientes resultados. (Dicho cuestionario se entregó a los 10 accionistas que integran esta Pyme, y las preguntas se anexan al final del presente trabajo)

La mayoría de las preguntas están enfocadas directamente a que sí la empresa cuenta con medidas para proteger al medio ambiente, si cuenta con algún reglamento del municipio para operar en materia ambiental, reconoce o registra los efectos medioambientales dentro de su información financiera, entre otras. El universo de la muestra se delimitó en esta empresa de manera específica dentro de la ciudad de México, debido a que se cuenta con información relativa a los reportes o informes financieros proporcionados por el administrador general de esta empresa.

También se hará uso del método comparativo para mostrar un balance financiero de la empresa sin considerar los efectos medioambientales y otro considerando las inversiones necesarias para proteger al medio ambiente.

\section{RESULTADOS}

Con el objeto de determinar o medir los niveles de percepción acerca de que si la empresa sujeta a estudio reconoce o registra un su contabilidad los efectos 
medioambientales, los dueños mencionaron en un $75 \%$ que no cuentan con un programa para proteger al medio ambiente, $20 \%$ argumentan que si cuentan con estas medidas y un $5 \%$ no sabe.

Figura 1. Medidas de protección para el medio ambiente

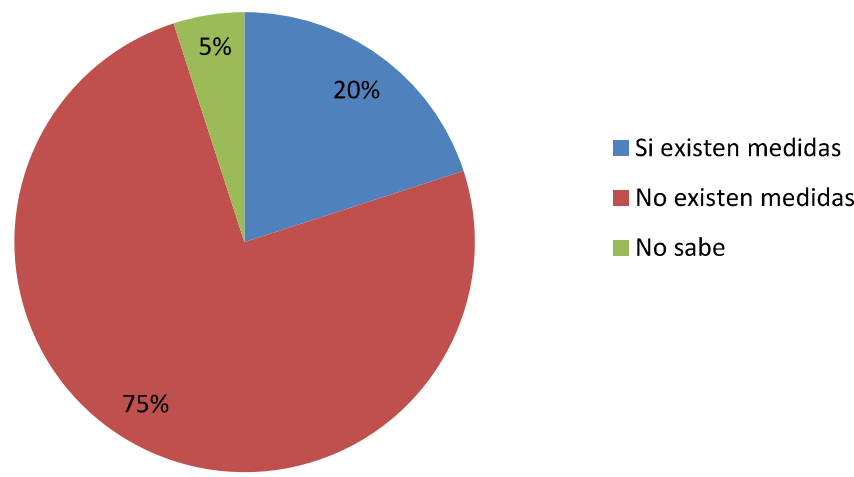

Fuente: Elaboración propia

Por otro lado, respecto de la pregunta si registran dentro de sus reportes o información financiera las inversiones, ganancias o pérdidas generadas por considerar o implementar medidas para proteger y cuidar al medio ambiente. El $90 \%$ de los dueños consideran que no, $5 \%$ dice que sí y el otro $5 \%$ no supieron.

Figura 2. Existencia de registros

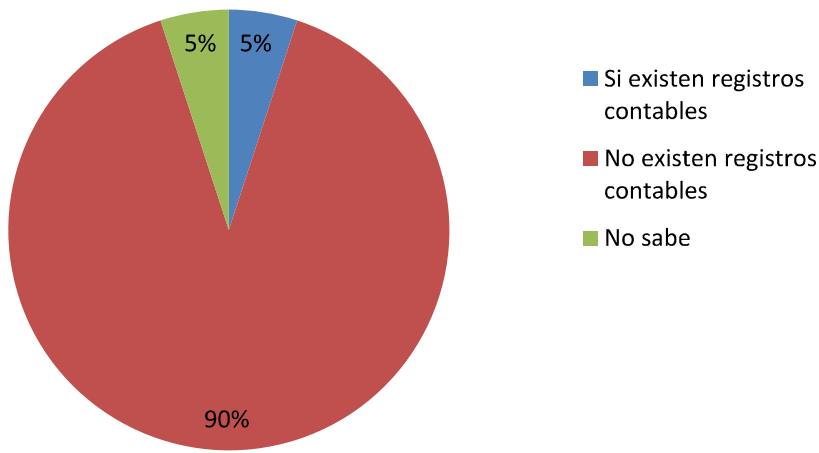

Fuente: Elaboración propia.

Se tomó como muestra estas dos preguntas, para poder inferir que los dueños de esta empresa, perciben que la forma de participar en la Responsabilidad Social Corporativa es a través de la sobrevivencia del negocio, obtener una rentabilidad financiera excelente, un crecimiento económico por encima de sus competidores, creación de nuevos empleos, ofrecer productos con una calidad superior. Todos estos factores son considerados como suficientes.

Por lo que respecta a la incorporación de los efectos medioambientales en los reportes o informes de carácter financiero, la empresa no lo considera relevante, ya que asigna mayor peso a las cuestiones económicas. Como parte del análisis de 
los resultados se solicitó a la administración de esta empresa nos proporcionara un Estado de posición Financiera, para constatar el no registro de los efectos medioambientales en dicho reporte.

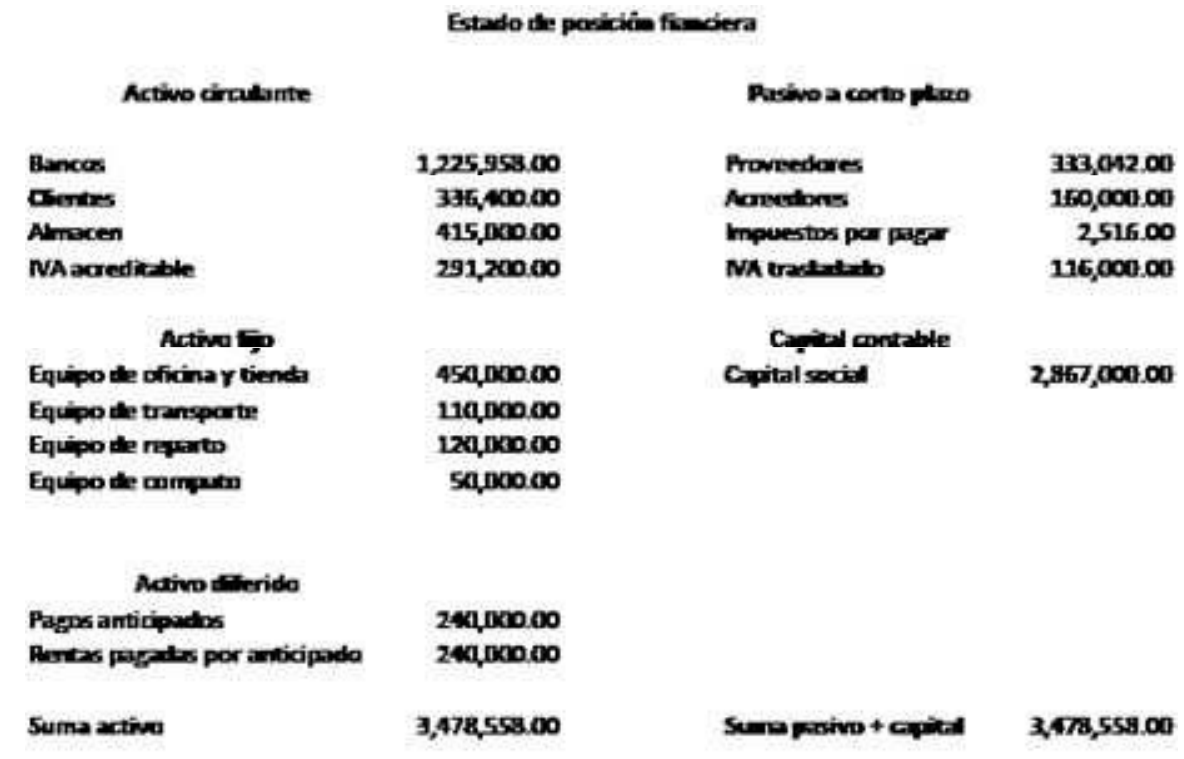

Propuesta de incorporación de los efectos medio ambientales en una contabilidad tradicional.

Como resultado de la investigación se hizo la siguiente propuesta hacia la dirección de la empresa para que registren sus inversiones en activos ambientales y por otro lado también se propone descontaminar los balances financieros al separar los costos ambientales que intervienen en su actividad económica como son el uso de energía eléctrica que no forme parte del costo de los productos, tener la posibilidad de invertir en paneles solares y cuantificar los posibles ahorros que se tendrían al reducir el consumo de energía eléctrica por el uso de energía solar dentro del estado de pérdidas y ganancias como un posible ingreso, recuperar el agua de lluvia para algunas actividades de la empresa como podría ser el uso en los inodoros, para regar jardines o a través de otras tecnologías para potabilizarla.

Ya que la inversión que se realiza a cierto tiempo en los recursos biológicos se vea reflejada como un beneficio y forma parte de un activo de la empresa, llegamos a la conclusión de que estos recursos se deberían de presentar dentro de la información financiera de la empresa.

Para el reconocimiento de los activos biológicos se propone que sea de la siguiente manera: 
Tabla 2. Clasificación de los activos biológicos

\begin{tabular}{|l|l|}
\hline Genero & Activo \\
\hline Grupo & Activo no circulante \\
\hline Rubro & Activos ambientales \\
\hline Cuenta & Activos biológicos \\
\hline Titulo & Deudora \\
\hline
\end{tabular}

\begin{tabular}{|c|l|c|l|}
\hline \multicolumn{3}{|c|}{ MOVIMIENTOS } \\
\hline NO. & \multicolumn{1}{|c|}{ CARGO } & NO. & \multicolumn{1}{|c|}{ ABONO } \\
\hline 1 & $\begin{array}{l}\text { DURANTE EL EJERCICIO } \\
\text { Por el costo de los activos } \\
\text { biológicos adquiridos. } \\
\text { Por el valor de los activos } \\
\text { biológicos recibidos en } \\
\text { donación o aportación } \\
\text { patrimonial. }\end{array}$ & 1 & $\begin{array}{l}\text { DURANTE EL EJERCICIO } \\
\text { Por el valor en libros de los activos biológicos } \\
\text { vendidos, transferidos dados de baja. }\end{array}$ \\
3 & $\begin{array}{l}\text { Por el valor de los activos } \\
\text { biológicos recibidos como pago } \\
\text { de algún deudor. } \\
\text { Por el valor de la actualización } \\
\text { del costo histórico de los } \\
\text { activos biológicos. }\end{array}$ & \\
\hline $\begin{array}{l}\text { SU SALDO REPRESENTA } \\
\text { El valor histórico de los activos biológicos así como la actualización. }\end{array}$ & \\
\hline
\end{tabular}

Fuente: Elaboración propia

Esta cuenta se presenta en el catálogo de cuentas de la empresa como sigue:

\begin{tabular}{|c|c|c|}
\hline Cuenta & Descripción de la cuenta & Saldo Final \\
\hline \multicolumn{3}{|c|}{ ACTIVO CIRCULANTE } \\
\hline $1120-000-000$ & BANCOS & $1,225,958.00$ \\
\hline $1120-001-000$ & BANMEX 123456789 & $1,225,958.00$ \\
\hline $1120-002-000$ & BANCO 2 & 0.00 \\
\hline $1140-000-000$ & CLIENTES & $336,400.00$ \\
\hline $1140-001-000$ & Emporio S.A. & $336,400.00$ \\
\hline $1150-000-000$ & ALMACÉN & $415,000.00$ \\
\hline $1150-001-000$ & Almacén "A" & $415,000.00$ \\
\hline $1190-000-000$ & IVA acreditable & $106,400.00$ \\
\hline $1191-000-000$ & IVA por acreditar & $184,800.00$ \\
\hline
\end{tabular}


EFECTOS MEDIOAMBIENTALES EN UNA CONTABILIDAD FINANCIERA DENTRO DE UNA PYME, ESTUDIO DE CASO

\begin{tabular}{|c|c|c|}
\hline \multicolumn{3}{|c|}{ ACTIVO FIJO } \\
\hline $1230-000-000$ & Equipo de oficina y tienda & $450,000.00$ \\
\hline $1240-000-000$ & Equipo de transporte & $110,000.00$ \\
\hline $1250-000-000$ & Equipo de reparto & $120,000.00$ \\
\hline $1260-000-000$ & Equipo de computo & $50,000.00$ \\
\hline \multicolumn{3}{|c|}{ ACTIVOS AMBIENTALES } \\
\hline $1310-000-000$ & RECURSOS BIOLOGICOS & $13,000.00$ \\
\hline \multicolumn{3}{|c|}{ ACTIVOS DIFERIDOS } \\
\hline $1310-000-000$ & PAGOS ANTICIPADOS & $240,000.00$ \\
\hline $1310-001-000$ & Rentas pagadas por anticipado & $240,000.00$ \\
\hline \multicolumn{3}{|c|}{ PASIVO CIRCULANTE } \\
\hline $2110-000-000$ & PROVEEDORES & $493,000.00$ \\
\hline $2110-001-000$ & Surtidora Mexicana S.A. de C.V. & $493,000.00$ \\
\hline $2120-000-000$ & ACREEDORES & $846,800.00$ \\
\hline $2120-001-000$ & Luis Campos Valverde & 0.00 \\
\hline $2120-002-000$ & Muebles para oficina S.A. & $522,000.00$ \\
\hline $2120-003-000$ & Distribuidora del norte S.A. & $139,200.00$ \\
\hline $2120-004-000$ & Soluciones integrales S.A. & $58,000.00$ \\
\hline $2120-005-000$ & FORD autos elegantes S.A. & $127,600.00$ \\
\hline $2140-000-000$ & IMPUESTOS POR PAGAR & $1,258.00$ \\
\hline $2140-001-000$ & ISPT & 820.00 \\
\hline $2140-002-000$ & IMSS & 438.00 \\
\hline $2150-000-000$ & IVA trasladado cobrado & $69,600.00$ \\
\hline $2151-000-000$ & IVA trasladado por cobrar & $46,400.00$ \\
\hline \multicolumn{3}{|c|}{ CAPITAL CONTABLE } \\
\hline $3100-000-000$ & CAPITAL SOCIAL & $1,500,000.00$ \\
\hline $3100-001-000$ & Armando Zarate Díaz & $500,000.00$ \\
\hline $3100-002-000$ & Carmen Sánchez Sarmiento & $500,000.00$ \\
\hline $3100-003-000$ & Adrián Jiménez López & $500,000.00$ \\
\hline \multicolumn{3}{|c|}{ CUENTAS DE RESULTADOS } \\
\hline $5000-000-000$ & VENTAS & $725,000.00$ \\
\hline $5100-000-000$ & Costo de venta & $435,000.00$ \\
\hline $5300-000-000$ & GASTOS DE ADMINISTRACION & $8,500.00$ \\
\hline
\end{tabular}

Fuente: Elaboración propia

Enseguida se presenta la propuesta de incorporación de los efectos medio ambientales en la contabilidad financiera de la empresa sujeto a estudio. 
Estado de pocición finedera

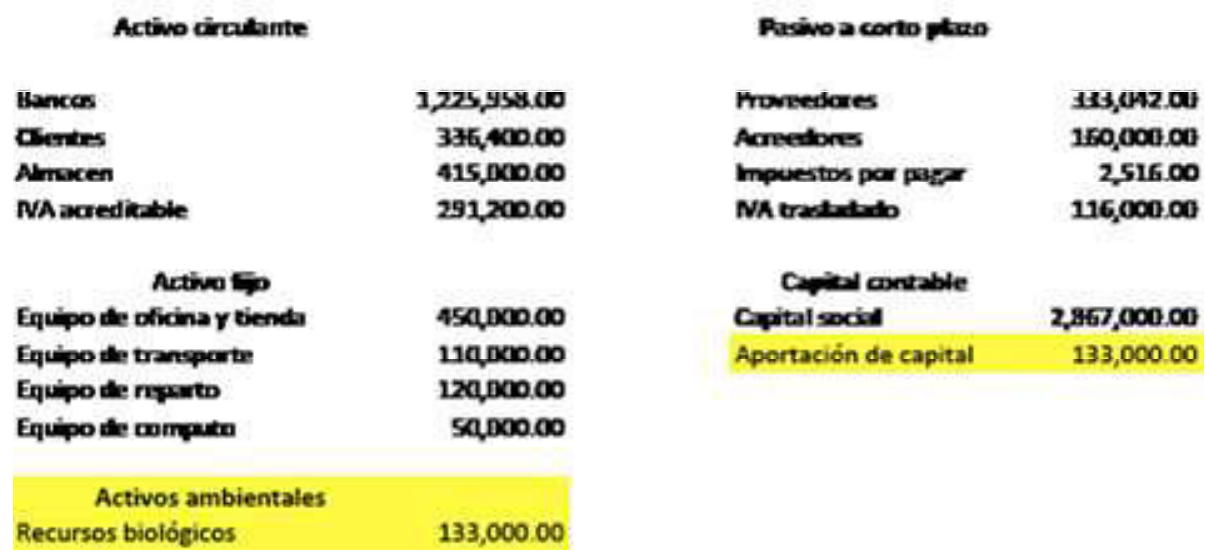

Propuesta de cómo medir el valor de una inversión inicial a futuro

Como todo activo se debe de actualizar el valor a partir del Monto Original de la Inversión, lo que al día de hoy se realiza con los activos de la empresa, ya sea, un incremento o decremento de su valor, en base a lo establecido dentro de la ley. En este caso, ¿cómo poder reconocer la actualización de un activo biológico? Si no existen parámetros para determinar dicho valor.

Para ello se propone tomar los factores como son:

- La inversión inicial en activos biológicos

- Número de personas que están interactuando en el entorno.

- El indicador de la Gaceta de Planeación Estratégica publicada el día 31 de octubre de 2012 por el Gobierno del Estado de México.

- Factor constante

Ya que hoy en día es una oportunidad dentro de los negocios considerar el impacto que se genera en el medioambiente, se busca resarcir el daño de estos, es por ello que se considera la inversión que se habrá de hacer en relaciones con las personas que interactúan en el entorno, para de esta manera poder conocer el beneficio que estos brindan y así valorar la actualización de la inversión en los recursos biológicos.

Pero sin perder de vista que no existe algún beneficio ya sea de manera económica o fiscal, se plantea el brindar algún beneficio fiscal o económico a aquellas empresas que inviertan en recursos biológicos que ayuden a resarcir el daño causado por las operaciones de estas.

\section{Auditoría ambiental (Seguimiento a la propuesta de implantación)}

Es el examen exhaustivo de los equipos y procesos que realiza una empresa, así como la contaminación y los riesgos de la misma, para evaluar el cumplimiento de las políticas ambientales establecidas y de los requerimientos normativos, Herrerías (2009).

Es el proceso de investigación realizado por auditor independiente, dirigido a 
determinar el grado de eficiencia empresarial, con relación al grado de satisfacción experimentado por la comunidad y su hábitat, señalando en su informe de auditoría a los agentes degradantes del medio ambiente y la magnitud de la degradación producida.

\section{CONCLUSIONES}

La contabilidad ambiental es un factor que se podría considerar dentro de los estados financieros con lo cual la empresa aumentaría el valor de esta y al mismo tiempo obtendrían un beneficio social.

Pero ésto no se toma en cuenta por parte de las empresas ya que se ve como un gasto y no como una inversión a largo plazo, que incluso genera un incremento en su valor a través del tiempo. Por lo que respecta a lo establecido en la hipótesis, podemos afirmar que la empresa sujeta a estudio, no reconoce los efectos del medio ambiente dentro de su contabilidad financiera, por lo que se hace necesario una propuesta de incorporación de estos efectos dentro de sus reportes financieros.

Si el problema se empieza a solucionar de manera interna en el gasto de la empresa con base en programas para combatir el impacto ambiental se beneficiarían a sí misma. Es por ello también que se hace necesario incorporar los efectos del medio ambiente en la contabilidad financiera de esta Pymes para incrementar su valor en libros, al registrar los beneficios de los activos intangibles.

Y ya que en el país se encuentra en un momento de transición esto podría tomar parte del cambio, ya que al imponer a las empresas que tomen acciones correctivas, el país tendría un alto valor en los recursos biológicos de la nación.

\section{ANEXO}

Tabla 3. Guía para responder el cuestionario ambiental del cliente

\begin{tabular}{|l|l|}
\hline & Escriba el nombre del accionista \\
\hline 1. & $\begin{array}{l}\text { Existen políticas ambientales dela empresa y en su caso estas deben ser aprobadas } \\
\text { por su Dirección General y en el caso de una sociedad anónima, por su Consejo de } \\
\text { Administración. }\end{array}$ \\
\hline 2. & $\begin{array}{l}\text { SI } \\
\text { La persona que ejerza las funciones de administrador ambiental de la empresa } \\
\text { asigna recursos para preservar el medio ambiente; si hubiese insuficientes recursos } \\
\text { asignados a una falta percibida de apoyo para las políticas y procedimientos de } \\
\text { control de riesgo ambiental, estas políticas y procedimientos podrían existir solo en } \\
\text { nombre pero no en sustancia. }\end{array}$ \\
\hline 3. & $\begin{array}{l}\text { SI } \\
\text { Los ejecutivos responsables deben observar las prácticas y la conducta del personal } \\
\text { de la empresa para formarse una opinión si tales prácticas y conductas cumplen con } \\
\text { los reglamentos ambientales (incluyendo leyes de salud y seguridad ocupacional) y si } \\
\text { es evidente que los procedimientos de control ambiental están siendo implantados. }\end{array}$ \\
\hline & SI $\quad$ NO \\
\hline
\end{tabular}


4. Las revisiones ambientales también se pueden denominar estudios, reportes, auditorias, etc., ambientales. Por lo general, se efectúan revisiones ambientales:

I. Para determinar el cumplimiento con los reglamentos ambientales (revisiones de cumplimiento).

II. Para determinar la efectividad de la administración que la empresa aplica a sus riesgos/problemas ambientales (revisiones de administración).

III. Para identificar los productos y equipos alternativos que son más "ambientalmente benignos" (revisiones de adquisiciones).

5. La falta de un certificado, permiso, etc., que se requiera podría indicar la presencia de un problema ambiental grave.

$$
\text { SI }
$$

6. A menudo las quejas del público es lo que detona la implantación de regulación por parte de agencias de gobierno, especialmente si la empresa se encuentra ubicada cerca de áreas ambientalmente sensitivas tales como:

- Parques o áreas de conservación.

- Lagos, ríos o arroyos.

- Montañas o áreas de escarpar.

- Bahías.

- Hábitat de especies en peligro de extinción.

- distritos especiales de agua subterránea.

- Lodazales.

- Áreas de bellos paisajes.

- Ex sitios de carbón, gas, terraplenes o botaderos de desechos, etc.

SI

7. Las quejas del público podrían ser un importante indicador de los problemas ambientales pasados, presentes o futuros que resulten de las actividades del cliente.

$$
\text { SI }
$$

8. Las notificaciones emitidas por autoridades supervisoras sobre problemas, podrían darse a través de aviso de infracción, multa, orden, prohibición, etc., tales ordenes deben ser revisadas ya que podrían indicar la existencia de un problema ambiental grave. Cuando el problema individualizado en la orden en que ha sido remediado, se requirió/obtuvo alguna aprobación reglamentaria respecto al trabajo resolutorio que se efectuó o efectuará.

SI

NO

9. Los costos estimados para remediar la situación/cierre/clausura y el posible impacto financiero del proceso legal/reglamentario puede ser relevante y deben ser factorizados dentro de proyecciones de flujo de caja relacionados al cliente. Estos costos deben incluir multas que hayan sido o puedan ser cargadas al cliente. 
\begin{tabular}{|l|l|}
10. & $\begin{array}{l}\text { Resuma los riesgos/problemas ambientales asociados al cliente, incluyendo } \\
\text { comentarios que no se hayan hecho en las preguntas anteriores. También haga sus } \\
\text { recomendaciones en caso que estos riesgos/problemas sean aceptables dentro del } \\
\text { contexto para incorporar estas medidas dentro de los informes o reportes financieros. }\end{array}$ \\
\hline
\end{tabular}

Fuente: Elaboración propia

\section{REFERENCIAS BIBLIOGRAFICAS}

Antonius, A. (1999). Exploração dos Recursos NaturaisFace à Sustentabilidade e Gestão Ambiental: UmaReflexão Teórico-Conceitual. Belém (Pa): NAEA.

Barraza, F. y Gómez, M. (2005). "Aproximación a un concepto de contabilidad ambiental" Bogotá, Colombia, Editorial Universidad cooperativa de Colombia, Facultad de Ciencias administrativas, económicas y contables.

Centro Mexicano para la Filantropía. (s.f). Recuperado 27/03/2013 de: http://www. cemefi.org/esr/_

Debernardo, H. y Hurtado, M. (2010). "Las Pymes. Principales causas de fracaso y cómo combatirlas", México, Grupo editorial ISEF

Enviroment Protection Agency US. (s.f). Recuperado 27/03/2013 de: http:/www.epa. gov/

Epstein, M. (2003). "El desempeño ambiental en la empresa”, Santa Fé de Bogotá, Lito Perla Impresores.

Gaceta del Gobierno del Estado de México. (31 de octubre de 2012). Manual para la planeación, programación y presupuestación municipal para el ejercicio fiscal 2013. Recuperado 28/03/2013 de: http://www.edomex.gob.mx/legistelfon/doc/pdf/ gct/2012/oct315.PDF

Gray, R. y Bobbington, J. (2006). “Contabilidad y Auditoría Ambiental”, Bogotá, Digiprint Editores.

Herrerías, E. (2009). "La problemática ambiental y su influencia en la contaduría". Recuperado 23/03/2013 de http://www.fca.unam.mx/capitulos/Unidad1.pdf

Herrerías, E. (2011). "Auditoría Ambiental". Recuperado 23/03/2013 de: http:// www.fca.unam.mx/capitulos/Unidad4.pdf

Herrerías, E. (2009). “Auditoría Ambiental”. Recuperado 13/06/2013 de: http://www. fca.unam.mx/capitulos/Unidad2.pdf

Horngreen, T., Sundem, L. y Elliot, J. (2000). "Introducción a la contabilidad financiera”, México, Pearson Educación de México, S.A. de C.V.

Iturria, D. (2012). "Costos ambientales"

Mercado, P. y García, P. (2013). "Sustentabilidad organizacional y responsabilidad social empresarial”. En Revista Emprendedores No. 143, pp. 48-51, UNAM, México. 
Normas Internacionales de Contabilidad. (s.f). Provisiones, Activos Contingentes y Pasivos Contingentes. Boletín Núm. 37. Recuperado 28/03/2013 de: http://www. normasinternacionalesdecontabilidad.es/nic/pdf/NIC37.pdf

Normatividad ambiental. (s.f). Recuperado 20/03/2013 de: http://www2.inecc.gob. $\mathrm{mx} /$ publicaciones/libros/621/normatividad.pdf

Yin, R. (1989), “Estudio de casos. Diseño y métodos”, México: Sage. 